\section{Meridional anisotropia in cyclofusion}

\section{STURLA KREKLING and SIGMUND BLIKA University of Trondheim, Trondheim, Norway}

By presenting a stereogram with half images consisting of a cross rotated by equal amounts, but in opposite directions, in the left and the right eye (Figure 1), O'Shea and Crassini (1982) observed that the near-vertical lines appeared fused (single), whereas the near-horizontal lines appeared diplopic. They concluded that their observation demonstrated the existence of a meridional anisotropia in the sensory fusional range for orientation-disparate stimuli (cyclofusion). This conclusion is open to criticism, since the apparent meridional difference that occurs during inspection of this stereogram might be due to the fact that the binocular system is disparity biased, that is, that the zero-orientation-disparity condition defined psychophysically is different from zero geometric disparity.

It is well known that the locus of points seen in the same (corresponding) visual direction by the left and the right eye is represented by a single line (vertical horopter) tilted in the median plane with its top away from the observer (e.g., Helmholtz, 1867; Tyler \& Scott, 1979). The tilted vertical horopter implies that binocular fusion is optimal when near-vertical images are rotated in a clockwise direction about the visual axis in the right eye and in a counterclockwise direction in the left eye. In accordance with Kertesz's (1973) sign convention for orientation disparity, this means that the vertical horopter is positively tilted. As shown by Cogan (1979), the angular magnitude of this tilt corresponds to about 2 deg of orientation disparity. Furthermore, the orientation-disparity bias of binocular correspondence is dependent on visualfield meridian with minimal bias near the horizontal meridian and maximum bias near the vertical visualfield meridian. This is illustrated by the curve in Figure 2, which was made on the basis of results reported by Volkmann (1863-1864). In his experiment, two lines slightly displaced to either side of the fixation point were presented dichoptically. The orientation of one line (reference) was set by the experimenter, and the observer adjusted the orientation of the other until both lines appeared parallel. This procedure was repeated for different orientations of the reference line. The orientation difference between lines matched for apparent orientation defines the disparity bias of cor-

This work was supported by the Norwegian Research Council for Science and the Humanities. Requests for reprints may be addressed to S. Blika, Department of Ophthalmology, University of Trondheim, N-7000 Trondheim, Norway.
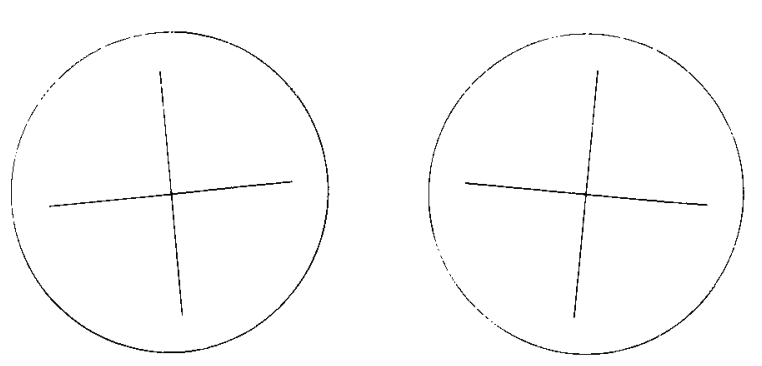

Figure 1. Dichoptic stimulus configuration. In the stereogram used by O'Shea and Crassini (1982), each monocular stimulus was rotated in opposite directions, right eye clockwise and left eye counterclockwise, about its center by 4 deg to produce an 8-deg positive orientation disparity. Most observers report that near-horizontal lines appear diplopic, whereas near-vertical lines are fused and appear as a single line tilted in depth. To obtain these effects without optical aids, the stereogram should be held at eye level at about $50 \mathrm{~cm}$ distance and be viewed with slightly converging visual axes (about $2 \mathrm{deg}$ ).

responding binocular meridians (Figure 2). Similar results were reported by Helmholtz (1867) and Nakayama (1977). This meridional skewness in the retinocortical mapping of corresponding binocular points implies that the effective disparity of a constant geometric orientation-disparate test line would also be meridionally dependent, since effective disparity would be equal to the difference between the

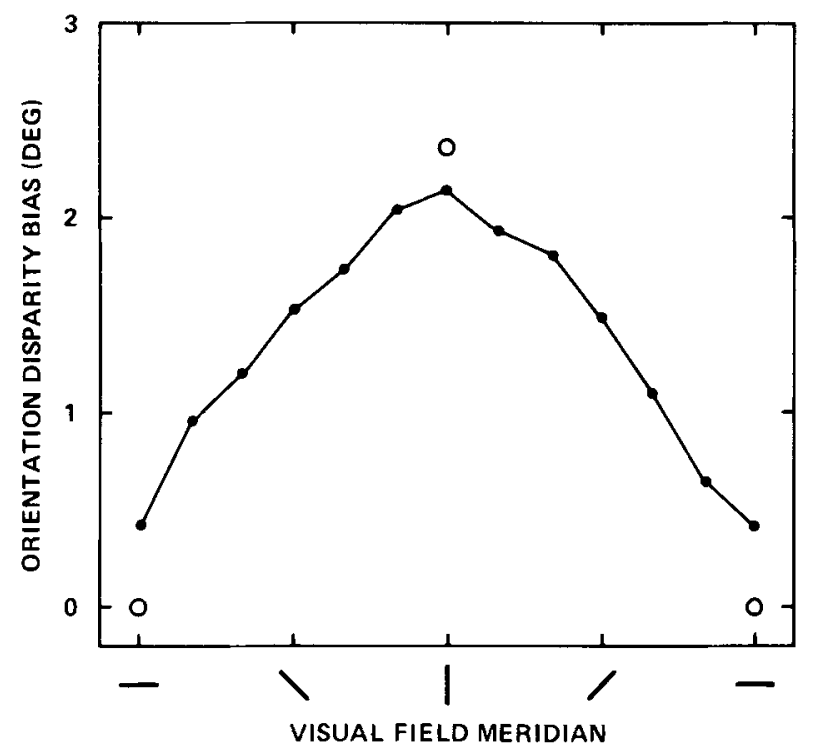

Figure 2. Meridional distribution of orientation-disparity bias plotted from data reported by Volkmann (1863-1864). Each data point represents the mean of 60 measurements. Open symbols represent data from Helmholtz (1867). 
disparity bias of the binocular system and the stimulus disparity. Assuming that the disparity bias at near-vertical visual-field meridians is about 2 deg positive, the effective disparity of an 8-deg positive orientation disparity would be equivalent to about 6 deg. This implies that in the stimulus configuration used by O'Shea and Crassini (1982), the effective disparity for near-vertical lines was smaller than it was for near-horizontal lines. Since fusion is more readily obtained for small disparities than for large disparities, near-vertical lines would appear to fuse more easily than near-horizontal lines even if the sensory fusional range were perfectly isotropic for all visualfield meridians.

In his comment on O'Shea and Crassini's (1982) results, Kertesz (1983) offered an entirely different interpretation. He assumed that the 8-deg orientationdisparity configuration (Figure 1) was beyond the range of the fusional response, but that the horizontal disparities in vertical lines are within the range of stereopsis. Consequently, both near-horizontal and near-vertical lines of the cross would appear diplopic. Since near-horizontal lines appear diplopic, whereas near vertical lines fuse and appear as a single line tilted in depth, Kertesz's (1983) suggestion would imply that the fusion of near-vertical orientations was caused by stereoptic mechanisms. If our analysis is correct, however, the fusional differences are due to the disparity bias of binocular corresponding points rather than to meridional differences in fusional range or stereoptic properties.

A meridional anisotropia in sensory fusional range might nevertheless exist. Crone and Leuridan (1973) investigated this problem by measuring the diplopia threshold for orientation disparity at near-vertical and horizontal meridians, and found that the fusional range was about three times wider at nearvertical than horizontal meridians. Similar results were reported by Ames (1926) and Beasley and Peckham (1936). In both these studies, test stimuli consisting of a pair of single lines were used. As shown by Hampton and Kertesz (1982), single-line stimuli do not evoke cyclofusional eye movements, which indicates that the meridional anisotropia in fusion is due to sensory processes. A meridional anisotropia in fusional range therefore suggests that the spatial extent of Panum's fusional areas increases with different rates as a function of eccentricity along vertical and horizontal visual-field meridians. This might possibly be related to a similar meridional difference in the disparity scatter (positional or orientational) of corresponding receptive fields in binocular neurons.

\section{REFERENCES}

Ames, A. Cyclophoria. American Journal of Physiological Optics, 1926, 7, 3-38.

Beasley, W. C., \& Peckham, R. H. An objective study of "cyclotorsion." Psychological Bulletin, 1936, 33, 741-742.

Cooan, A. I. The relationship between the apparent vertical and the vertical horopter. Vision Research, 1979, 19, 655-665.

Crone, R. A., \& Leuridan, O. M. A. Tolerance for aniseikonia. II. Determination based on the amplitude of cyclofusion. Albrecht von Graefes Archiv für klinische und experimentelle Ophthalmologie, 1973, 188, 17-22.

Hampton, D. R., \& Kertesz, A. E. Human response to cyclofusional stimuli containing depth cues. American Journal of Optometry and Physiological Optics, 1982, 59, 21-27.

HeLmholtz, H. von Handbuch der physiologischen Optik. Leipzig: Leopold Voss, 1867.

Kertesz, A. E. Disparity detection within Panum's fusional areas. Vision Research, 1973, 13, 1537-1543.

Kertesz, A. E. Cyclofusion and stereopsis. Perception \& Psychophysics, 1983, 33, 99-101.

Naxayama, K. Geometric and physiological aspects of depth perception. Proceedings of the Society of Photo-Optical Instrumentation Engineers, 1977, 120, $2-9$.

O'Shea, R. P., \& Crassini, B. The dependence of cyclofusion on orientation. Perception \& Psychophysics, 1982, 32, 195-196.

Tyler, C. W., \& ScotT, A. B. Binocular vision. In R. E. Records (Ed.), Physiology of the human eye and visual system. New York: Harper \& Row, 1979.

Volkmann, A. W. Physiologische Untersuchungen im Gebiete der Optik. Leipzig: Breitkopf \& Härtel, 1863-1864.

(Manuscript received May 9, 1983; accepted for publication May 31, 1983.) 